\title{
Front Matter: Volume 8616
}

, "Front Matter: Volume 8616," Proc. SPIE 8616, MOEMS and Miniaturized Systems XII, 861601 (13 March 2013); doi: 10.1117/12.2022906

SPIE. Event: SPIE MOEMS-MEMS, 2013, San Francisco, California, United States 


\title{
PROCEEDINGS OF SPIE
}

\section{MOEMS and Miniaturized Systems XII}

\author{
Wibool Piyawattanametha \\ Yong-Hwa Park \\ Editors
}

\section{4-6 February 2013}

San Francisco, California, United States

Sponsored by

SPIE

Cosponsored by

Bridger Photonics, Inc. (United States)

VUZIX Corporation (United States)

Supported by

Fraunhofer Institute for Photonic Microsystems (Germany)

Published by

SPIE 
The papers included in this volume were part of the technical conference cited on the cover and title page. Papers were selected and subject to review by the editors and conference program committee. Some conference presentations may not be available for publication. The papers published in these proceedings reflect the work and thoughts of the authors and are published herein as submitted. The publisher is not responsible for the validity of the information or for any outcomes resulting from reliance thereon.

Please use the following format to cite material from this book:

Author(s), "Title of Paper," in MOEMS and Miniaturized Systems XII, edited by Wibool Piyawattanametha, Yong-Hwa Park, Proceedings of SPIE Vol. 8616 (SPIE, Bellingham, WA, 2013) Article CID Number.

ISSN: 0277-786X

ISBN: 9780819493859

Published by

SPIE

P.O. Box 10, Bellingham, Washington 98227-0010 USA

Telephone +1 3606763290 (Pacific Time) · Fax +1 3606471445

SPIE.org

Copyright @ 2013, Society of Photo-Optical Instrumentation Engineers.

Copying of material in this book for internal or personal use, or for the internal or personal use of specific clients, beyond the fair use provisions granted by the U.S. Copyright Law is authorized by SPIE subject to payment of copying fees. The Transactional Reporting Service base fee for this volume is $\$ 18.00$ per article (or portion thereof), which should be paid directly to the Copyright Clearance Center (CCC), 222 Rosewood Drive, Danvers, MA 01923. Payment may also be made electronically through CCC Online at copyright.com. Other copying for republication, resale, advertising or promotion, or any form of systematic or multiple reproduction of any material in this book is prohibited except with permission in writing from the publisher. The CCC fee code is 0277-786X/13/\$18.00.

Printed in the United States of America.

Publication of record for individual papers is online in the SPIE Digital Library.

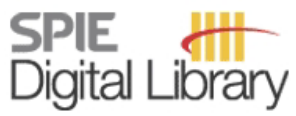

SPIEDigitalLibrary.org

Paper Numbering: Proceedings of SPIE follow an e-First publication model, with papers published first online and then in print and on CD-ROM. Papers are published as they are submitted and meet publication criteria. A unique, consistent, permanent citation identifier (CID) number is assigned to each article at the time of the first publication. Utilization of CIDs allows articles to be fully citable as soon as they are published online, and connects the same identifier to all online, print, and electronic versions of the publication. SPIE uses a six-digit CID article numbering system in which:

- The first four digits correspond to the SPIE volume number.

- The last two digits indicate publication order within the volume using a Base 36 numbering

system employing both numerals and letters. These two-number sets start with 00, 01, 02, 03, 04, $05,06,07,08,09,0 A, 0 B \ldots$. 0Z, followed by 10-1Z, 20-2Z, etc.

The CID Number appears on each page of the manuscript. The complete citation is used on the first page, and an abbreviated version on subsequent pages. Numbers in the index correspond to the last two digits of the six-digit CID Number. 


\section{Contents}

ix Conference Committee

MOEMS-MEMS PLENARY SESSION

861602 Towards future systems with nano-optics contributions (Plenary Paper) [8616-101]

B. Kaminska, Simon Fraser Univ. (Canada) and NanoTech Security Corp. (Canada); M. Najiminaini, Simon Fraser Univ. (Canada), Lawson Health Research Institute (Canada), and The Univ. of Western Ontario (Canada); Y. Chuo, C. Landrock, B. Omrane, Simon Fraser Univ. (Canada) and NanoTech Security Corp. (Canada); J. J. L. Carson, Lawson Health Research Institute (Canada) and The Univ. of Western Ontario (Canada)

861603 MOEMS pressure sensors for geothermal well monitoring (Plenary Paper) [8616-102] W. Challener, S. Palit, General Electric Global Research (United States); R. Jones, L. Airey, R. Craddock, General Electric Measurement and Control (United Kingdom); A. Knobloch, General Electric Global Research (United States)

\section{SESSION 1 MICROSCOPY: JOINT SESSION WITH CONFERENCES 8616 AND 8575}

861604 Optical probe design with extended depth-of-focus for optical coherence microscopy and optical coherence tomography [8616-1]

S. Lee, M. Choi, E. Lee, K.-D. Jung, J. Chang, W. Kim, Samsung Advanced Institute of Technology (Korea, Republic of)

861605 3D micro-optical lens scanner made by multi-wafer bonding technology [8616-2] S. Bargiel, C. Gorecki, M. Barański, N. Passilly, FEMTO-ST (France); M. Wiemer, C. Jia, J. Frömel, Fraunhofer-Institut für Elektronische Nanosysteme (Germany)

861606 Electrostatic MEMS resonating micro-polygonal scanner for circumferential endoscopic bio-imaging [8616-3]

X. MU, National Univ. of Singapore (Singapore) and A*STAR Institute of Microelectronics (Singapore); G. Zhou, National Univ. of Singapore (Singapore); H. YU, J. M.-L. Tsai, A*STAR Institute of Materials Research and Engineering (Singapore); W. K. Neo, A. S. Kumar,

F. S. Chau, National Univ. of Singapore (Singapore)

861607 A water-immersible two-axis scanning mirror microsystem for ultrasound and photoacoustic microscopic imaging applications [8616-4]

C.-H. Huang, Texas A\&M Univ. (United States); J. Yao, L. V. Wang, Washington Univ. in St. Louis (United States); J. Zou, Texas A\&M Univ. (United States) 
8616 OA Optical scanners based on thermo-optical tuning of an integrated-optical waveguide mode [8616-7]

E. Markweg, M. Hoffmann, Technische Univ. Ilmenau (Germany)

8616 0C Resonant biaxial 7-mm MEMS mirror for omnidirectional scanning (Best Paper Award) [8616-9]

U. Hofmann, Fraunhofer-Institut für Siliziumtechnologie (Germany); M. Aikio, VTT Technical Research Ctr. of Finland (Finland); J. Janes, F. Senger, V. Stenchly, M. Weiss, H.-J. Quenzer,

B. Wagner, W. Benecke, Fraunhofer-Institut für Siliziumtechnologie (Germany)

\section{SESSION $3 \quad$ MICROSCANNER II}

8616 OD Position sensing and tracking with quasistatic MEMS mirrors (Invited Paper) [8616-11]

S. Richter, M. Stutz, A. Gratzke, Y. Schleitzer, G. Krampert, F. Hoeller, U. Wolf, L. Riedel,

D. Doering, Carl Zeiss AG (Germany)

$86160 \mathrm{O} \quad$ Aluminum nitride supported 1D micromirror with static rotation angle $>11^{\circ}$ [8616-12]

S. Weinberger, M. Hoffmann, Technische Univ. Ilmenau (Germany)

8616 OF Wide steering angle microscanner based on curved surface (Best Student Paper Award) [8616-13]

Y. Sabry, Univ. Paris-Est (France) and Si-Ware Systems (Egypt); D. Khalil, Si-Ware Systems (Egypt) and Ain-Shams Univ. (Egypt); B. Saadany, Si-Ware Systems (Egypt); T. Bourounia, Univ. Paris-Est (France)

$86160 G$ SOI based electromagnetic MEMS scanners and applications in laser systems [8616-14] G. Brown, R. Baver, W. Lubeigt, D. Uttamchandani, The Univ. of Strathclyde (United Kingdom)

SESSION 4 SPATIAL LIGHT MODULATOR: JOINT SESSION WITH CONFERENCES 8616 AND 8618

$8616 \mathrm{OH} \quad$ Arrayed beam steering device for advanced 3D displays (Invited Paper) [8616-15] J. Bae, Y.-S. Choi, K. Choi, Y. Kim, Y. Kwon, H. Song, E. Kim, Samsung Advanced Institute of Technology (Korea, Republic of); S. Choi, J. Lee, Seoul National Univ. (Korea, Republic of); S. Lee, Samsung Advanced Institute of Technology (Korea, Republic of)

8616 Ol 2D electrostatic micromirror array with high field factor for high-power application [8616-16]

S. Lani, D. Bayat, Y. Pétremand, Ctr. Suisse d'Electronique et de Microtechnique SA (Switzerland)

8616 J Jabrication of vertical moving micro-optical switch for display applications [8616-17] D. Shim, W. Kim, H. Choi, Samsung Advanced Institute of Technology (Korea, Republic of) 
8616 OK Development of a fully programmable MEMS diffraction grating [8616-18]

F. Zamkotsian, Lab. d'Astrophysique de Marseille (France); B. Timotijevic, Ctr. Suisse d'Electronique et de Microtechnique SA (Switzerland); R. Lockhart, Ecole Polytechnique Fédérale de Lausanne (Switzerland); R. P. Stanley, Ctr. Suisse d'Electronique et de Microtechnique SA (Switzerland); P. Lanzoni, Lab. d'Astrophysique de Marseille (France); M. Luetzelschwab, Ctr. Suisse d'Electronique et de Microtechnique SA (Switzerland); M. Canonica, Massachusetts Institute of Technology (United States); W. Noel, Ecole Polytechnique Fédérale de Lausanne (Switzerland); M. Tormen, Ctr. Suisse d'Electronique et de Microtechnique SA (Switzerland)

\section{SESSION 5 MICROSPECTROMETER AND OPTICAL FILTERS}

$8616 \mathrm{OL}$ Design and characterization of a hybrid-integrated MEMS scanning grating spectrometer (Invited Paper) [8616-19]

H. Grüger, J. Knobbe, T. Pügner, H. Schenk, Fraunhofer-Institut für Photonische Mikrosysteme (Germany)

$86160 \mathrm{M}$ SWIFTS: a groundbreaking integrated technology for high-performance spectroscopy and optical sensors (Invited Paper) [8616-20]

C. Bonneville, Resolution Spectra Systems (France); F. Thomas, Resolution Spectra Systems (France) and Institut de Planétologie et d'Astrophysique de Grenoble (France);

M. de Mengin Poirier, E. Le Coarer, Institut de Planétologie et d'Astrophysique de Grenoble (France); P. Benech, IMEP-LAHC (France); T. Gonthiez, Resolution Spectra Systems (France): A. Morand, IMEP-LAHC (France); O. Coutant, Institut des Sciences de la Terre (France); E. Morino, R. Puget, B. Martin, Resolution Spectra Systems (France)

$86160 \mathrm{~N}$ Fabrication and evaluation of a $500 \mathrm{~W}$ cladding-light stripper [8616-21]

A. Kliner, Friedrich-Schiller-Univ. Jena (Germany) and Fraunhofer-Institut für Angewandte Optik und Feinmechanik (Germany); K.-C. Hou, JDSU (United States); M. Plötner, Ch. Hupel, Th. Stelzner, T. Schreiber, R. Eberhardt, Fraunhofer-Institut für Angewandte Optik und Feinmechanik (Germany); A. Tünnermann, Friedrich-Schiller-Univ. Jena (Germany) and Fraunhofer-Institut für Angewandte Optik und Feinmechanik (Germany)

861600 Spatial beam splitting for fully integrated MEMS interferometer [8616-22] K. Khirallah, I. Ramsis, M. Serry, The American Univ. in Cairo (Egypt); M. Swillam, The American Univ. in Cairo (Egypt) and The Univ. of Toronto (Canada); S. Sedky, Zewail City of Science and Technology (Egypt)

8616 OP In-plane diffraction loss free optical cavity using coated optical fiber and silicon micromachined spherical mirror [8616-23]

Y. Sabry, Univ. Paris-Est (France) and Si-Ware Systems (Egypt); T. Bourounia, Univ. Paris-Est (France); B. Saadany, Si-Ware Systems (Egypt); D. Khalil, Si-Ware Systems (Egypt) and AinShams Univ. (Egypt)

$86160 Q \quad$ A tunable split-ladder photonic crystal cavity by nanoelectromechanical actuator [8616-24]

F. Tian, G. Zhou, F. S. Chau, National Univ. of Singapore (Singapore); J. Deng, R. Akkipeddi, X. Tang, S. L. Teo, Y. C. Loke, A*STAR Institute of Materials Research and Engineering (Singapore) 
8616 OR Compact holographic printer using RGB waveguide holographic optical elements (Invited Paper) [8616-25]

K. P. Pyun, C. Choi, Samsung Advanced Institute of Technology (Korea, Republic of);

A. V. Morozov, Samsung Moscow Research Ctr. (Russian Federation); S. Kim, J. An,

Samsung Advanced Institute of Technology (Korea, Republic of)

8616 OT Innovative approach to high stroke electrostatic actuators [8616-27]

N. Lange, Friedrich-Schiller-Univ. Jena (Germany) and Fraunhofer-Institut für Angewandte Optik und Feinmechanik (Germany); F. Wippermann, E. Beckert, R. Eberhardt, FraunhoferInstitut für Angewandte Optik und Feinmechanik (Germany); A. Tünnermann, FriedrichSchiller-Univ. Jena (Germany) and Fraunhofer-Institut für Angewandte Optik und Feinmechanik (Germany)

$86160 \mathrm{U}$ Tunable MEMS diffraction gratings with improved displacement profile of the fixed-fixed beams [8616-28]

V. G. Suresh, S. Bhattacharya, N. Dasgupta, Indian Institute of Technology Madras (India)

8616 OV Optimization of biogas production using MEMS based near infrared inline-sensor [8616-30] R. Saupe, T. Seider, Fraunhofer-Institut für Elektronische Nanosysteme (Germany); V. Stock, TQ-Systems GmbH (Germany); O. Kujawski, LimnoTec GmbH (Germany); T. Otto,

T. Gessner, Fraunhofer-Institut für Elektronische Nanosysteme (Germany)

\section{SESSION 7 MOEMS COMPONENTS AND SYSTEMS II}

$86160 \mathrm{X}$ InGaAsP optical device integration on SOI platform by $\mathrm{Ar} / \mathrm{O}_{2}$ plasma assisted bonding [8616-32]

A. Higo, Tohoku Univ. (Japan); L. Li, E. Higurashi, M. Sugiyama, Y. Nakano, The Univ. of Tokyo (Japan)

8616 OY A new fabrication method for nano-gratings based on the high flexibility of PDMS [8616-33] M. Cui, Y. Zhang, B. Zhang, W. Wang, North Univ. of China (China)

$86160 Z$ Development of a focusing micromirror device with an in-plane stress relief structure in SOI technology [8616-34]

W. Kronast, U. Mescheder, B. Müller, R. Huster, Hochschule Furtwangen Univ. (Germany)

\section{SESSION 8 MICROLENSES AND MICROLENS ARRAYS}

861610 Development of adaptive liquid microlenses and microlens arrays [8616-35]

S. R. Berry, J. B. Stewart, T. A. Thorsen, I. Guha, MIT Lincoln Lab. (United States)

861611 Tunable cylindrical microlenses based on aluminum nitride membranes [8616-37] S. Leopold, D. Paetz, Technische Univ. Ilmenau (Germany); F. Knoebber, O. Ambacher, Fraunhofer-Institut für Angewandte Festkörperphysik (Germany); S. Sinzinger, M. Hoffmann, Technische Univ. IImenau (Germany) 
861612 Liquid lens based on electrowetting: actual developments on larger aperture and multiple electrodes design for image stabilization or beam steering [8616-38]

B. Berge, J. Broutin, H. Gaton, G. Malet, E. Simon, F. Thieblemont, Varioptic-A BU of Parrot SA (France)

861613 The fabrication of out of plane aspherical microlens arrays [8616-39]

Y. Zhang, W. Wang, North Univ. of China (China)

SESSION 9 IMAGING

861614 MOEMS-based time-of-flight camera for 3D video capturing (Invited Paper) [8616-40] J.-W. You, Y.-H. Park, Y.-C. Cho, C.-Y. Park, H. Yoon, S.-H. Lee, S.-W. Lee, Samsung Advanced Institute of Technology (Korea, Republic of)

861615 Simultaneous multispectral imaging using lenslet arrays [8616-41] M. Hinnrichs, Pacific Advanced Technology, Inc. (United States); J. Jensen, U.S. Army Edgewood Chemical Biological Ctr. (United States)

861616 Batch fabrication of micro-optical sensing and imaging devices [8616-42] F. C. Wippermann, A. Reimann, A. Oelschläger, P. Dannberg, Fraunhofer-Institut für Angewandte Optik und Feinmechanik (Germany); F. Blöhbaum, SICK AG (Germany); C. Koburg, T. Köhler, Continental Automotive GmbH (Germany)

861617 Diffraction and photometric limits in today's miniature digital camera systems [8616-43] A. Brückner, Fraunhofer-Institut für Angewandte Optik und Feinmechanik (Germany); M. Schöberl, Fraunhofer-Institut für Integrierte Schaltungen (Germany)

861618 Optical MEMS in space instruments for Earth observation and astronomy [8616-44] F. Zamkotsian, Lab d'Astrophysique de Marseille, CNRS (France); A. Liotard, Thales Alenia Space (France); P. Lanzoni, Lab d'Astrophysique de Marseille, CNRS (France); T. Viard, Thales Alenia Space (France)

\section{POSTER SESSION}

861619 Closed-loop control driver for quasi-static MOEMS mirrors [8616-45]

A. Tortschanoff, D. Holzmann, M. Lenzhofer, Carinthian Tech Research AG (Austria); T. Sandner, Fraunhofer-Institut für Photonische Mikrosysteme (Germany); A. Kenda, Carinthian Tech Research AG (Austria)

Author Index 
Proc. of SPIE Vol. $8616861601-8$

Downloaded From: https://www.spiedigitallibrary.org/conference-proceedings-of-spie on 25 Apr 2023 Terms of Use: https://www.spiedigitallibrary.org/terms-of-use 


\section{Conference Committee}

Symposium Chair

Harald Schenk, Fraunhofer Institute for Photonic Microsystems

(Germany)

Symposium Cochair

David L. Dickensheets, Montana State University (United States)

Conference Chairs

Wibool Piyawattanametha, NECTEC (Thailand) and Chulalongkorn University (Thailand)

Yong-Hwa Park, Samsung Advanced Institute of Technology

(Korea, Republic of)

Conference Program Committee

Wyatt O. Davis, MicroVision, Inc. (United States)

David L. Dickensheets, Montana State University (United States)

Jean-Christophe Eloy, Yole Développement (France)

Sonia M. García-Blanco, Universiteit Twente (Netherlands)

Jason C. Heikenfeld, University of Cincinnati (United States)

II-Woong Jung, Argonne National Laboratory (United States)

Jonathan T. C. Liu, Stony Brook University (United States)

Veljko Milanovic, Mirrorcle Technologies, Inc. (United States)

Harald Schenk, Fraunhofer Institute for Photonic Microsystems

(Germany)

Jason B. Stewart, MIT Lincoln Laboratory (United States)

Wanjun Wang, Lovisiana State University (United States)

Guangya Zhou, National University of Singapore (Singapore)

\section{Session Chairs}

1 Microscopy: Joint Session with Conferences 8616 and 8575

David L. Dickensheets, Montana State University (United States)

2 Microscanner I

Wibool Piyawattanametha, NECTEC (Thailand) and Chulalongkorn University (Thailand) 
3 Microscanner II

Yong-Hwa Park, Samsung Advanced Institute of Technology (Korea, Republic of)

4 Spatial Light Modulator: Joint Session with Conferences 8616 and 8618 Harald Schenk, Fraunhofer Institute for Photonic Microsystems (Germany)

5 Microspectrometer and Optical Filters

Guangya Zhou, National University of Singapore (Singapore)

6 MOEMS Components and Systems I

Jason B. Stewart, MIT Lincoln Laboratory (United States)

7 MOEMS Components and Systems II

II-Woong Jung, Argonne National Laboratory (United States)

8 Microlenses and Microlens Arrays

Veljko Milanovic, Mirrorcle Technologies, Inc. (United States)

9 Imaging

Wyatt O. Davis, MicroVision, Inc. (United States) 